\title{
Oscillator Noise Analysis
}

Alper Demir

Citation: AIP Conference Proceedings 780, 499 (2005); doi: 10.1063/1.2036801

View online: http://dx.doi.org/10.1063/1.2036801

View Table of Contents: http://aip.scitation.org/toc/apc/780/1

Published by the American Institute of Physics 


\title{
Oscillator Noise Analysis
}

\author{
Alper Demir ${ }^{1}$ \\ Department of Electrical \& Electronics Engineering, Koc University, Istanbul, Turkey
}

\begin{abstract}
Oscillators are key components of many kinds of systems, particularly electronic and opto-electronic systems. Undesired perturbations, i.e. noise, that exist in practical systems adversely affect the spectral and timing properties of the signals generated by oscillators resulting in phase noise and timing jitter. These are key performance limiting factors, being major contributors to biterror-rate (BER) of RF and optical communication systems, and creating synchronization problems in clocked and sampled-data electronic systems. In noise analysis for oscillators, the key is figuring out how the various disturbances and noise sources in the oscillator end up as phase fluctuations. In doing so, one first computes transfer functions from the noise sources to the oscillator phase, or the sensitivity of the oscillator phase to these noise sources. In this paper, we first provide a discussion explaining the origins and the proper definition of this transfer or sensitivity function, followed by a critical review of the various numerical techniques for its computation that have been proposed by various authors over the past fifteen years.
\end{abstract}

Keywords: oscillators, noise, Floquet theory, phase noise, timing jitter

PACS: $84.30 . \mathrm{Bv}, 85.40 . \mathrm{Qx}, 84.30 . \mathrm{Ng}, 07.50 . \mathrm{Hp}$

\section{PROBLEM DEFINITION}

In oscillator noise analysis, the key problem is the determination of how exactly the various disturbances and noise sources in the oscillator cause phase fluctuations. For the solution of this problem, one defines and computes transfer functions (in a loose sense) or sensitivities, from all of the noise sources to the oscillator phase, that govern and quantify the conversion of the disturbances and noise into phase fluctuations.

\section{PHASE NOISE SENSITIVITIES}

In the literature, authors have used various names for the phase noise sensitivities mentioned above, the Floquet Vector (FV) [1, 2], the Impulse Sensitivity Function (ISF) [3], and the Perturbation Projection Vector (PPV) [4]. The terms Floquet Vector and Impulse Sensitivity Function were inspired by the theory or the techniques the authors have used to define or compute the sensitivities. The term Perturbation Projection Vector was chosen considering how the phase noise sensitivities are used in oscillator noise analysis. It can be shown that the definitions for all of the above (FV, ISF and PPV) are, deep down, theoretically equivalent. On the other hand, the (numerical) algorithms that are used for computing the phase noise sensitivities are quite different from each other in the various approaches mentioned above.

\section{FLOQUET VECTOR AND FLOQUET THEORY}

From this point on, we will refer to the collection of the phase noise sensitivities as the Floquet Vector (FV) to honor the French mathematician G. Floquet for having had developed the Floquet Theory, a very simple yet elegant and practically useful theory that forms the basis for the analysis of linear periodically time-varying (LPTV) systems. Without delving into the details and equations, Floquet Theory is essentially an eigen-

\footnotetext{
${ }^{1}$ Sponsored by the Turkish Academy of Sciences GEBIP program and a TUBITAK Career Award.
} 
decomposed solution analysis for LPTV systems, an elegant and simple generalization of the widely utilized eigen-decomposed analysis technique for linear time-invariant (LTI) systems. In eigen-decomposed analysis, the response or the solution of the (linear) system is expressed as a summation of the eigen-modes of the system, from undamped to highly-damped modes corresponding to real or pair of complex-conjugate poles (i.e., eigenvalues) resulting in fast, slow, decaying, growing, persistent, or possibly oscillatory behavior.

\section{ORIGINS OF THE FLOQUET VECTOR}

The discussion and definition of a transfer or sensitivity function from the noise sources to the oscillator phase, to the best of our knowledge, appeared first in Kaertner's work $[5,6]$. It was Kaertner who first perceptively observed that the disturbances in an oscillator result in both orbital deviations (amplitude deviations in a loose sense) that "eventually" die away and phase fluctuations that persist indefinitely even if the disturbances disappear. He then used the mechanics of the Floquet Theory to decompose the response of an oscillator to disturbances into two sets of eigen-modes, the first containing all of the modes that eventually decay away and the second set comprised of a single mode that persists indefinitely.

Without delving into equations, we now describe how the FV mentioned above was defined and computed by Kaertner in [6]. When a stable nonlinear oscillator is linearized around its periodic steady-state solution (PSSS), one obtains an LPTV system. When an eigen-decomposed analysis for this LPTV system is performed using Floquet Theory, it can be shown that one particular eigen-mode has to be non-decaying. Even though this eigen-mode is persistent, it does not grow without bound. The existence of a nondecaying mode, in essence, is equivalent to the steady-state oscillation condition. Any oscillator which supports a periodic, stable, steady-state solution has to have one nondecaying, persistent but non-growing mode associated with the LPTV system obtained by linearization. The time-derivative of the periodic steady-state, large-signal solution of the nonlinear oscillator (which is also periodic and persistent) turns out to be the persistent mode of the LPTV system obtained by linearization. Thus, once the largesignal solution for the nonlinear oscillator is computed, the persistent mode of the LPTV system can easily be computed by differentiation in time. In oscillator phase noise analysis, the key is the decomposition of the disturbances and noise into their modal components, one component that is aligned with the persistent eigen-mode discussed above, and the rest aligned with the eigen-modes which decay away eventually.

The eigen-mode analysis for LPTV (and LTI) systems can be cast as a matrix eigenvalue/eigenvector problem, by using Fourier series representations for the eigen-modes with a harmonic balance formulation, or through time discretization in connection with a finite-difference formulation. Then, the eigen-modes for a linear system become equivalent to the eigenvectors of a matrix and they form a basis set that span the space of all possible solutions. If the eigen-modes are orthogonal to each other, one can easily perform the modal decomposition mentioned above using orthogonal projection and extract the components of the disturbances aligned with the persistent mode, without the need to compute the other decaying eigen-modes. The eigen-modes of a linear system are orthogonal to each other if the system is self-adjoint, or equivalently, when cast as a matrix eigenvalue/eigenvector problem, if the matrix is symmetric. Unfortunately, the LPTV system or circuit obtained by the linearization of an oscillator, in general, is not self-adjoint. It turns out, however, that the eigen-modes of the adjoint system are biorthogonal to the eigen-modes of the system itself. The adjoint operator for linear systems or circuits is (somewhat loosely) analogous to the transpose operator for matrices. The non-decaying mode of the adjoint of the LPTV system obtained by linearization is orthogonal to all of the decaying modes of the system itself, and hence, it can be 
used in an orthogonal projection operation to extract the component of the disturbances and noise that are aligned with the persistent mode for the LPTV system. To extract this component out of a composite noise response, one uses the persistent mode of the adjoint system, not the system itself. The composite response is a summation (linear combination) of contributions from all eigen-modes. If one computes the inner product of the persistent adjoint mode with the composite response, the result is a quantification of how much of the persistent mode for the system itself is in the composite response. This is so, because the inner product of the adjoint mode with all of the other modes is zero due to bi-orthogonality. Thus, the non-decaying mode of the adjoint of the LPTV system obtained by the linearization of the oscillator around the PSSS yields the FV that lies at the heart of oscillator noise analysis.

\section{NUMERICAL TECHNIQUES FOR THE FLOQUET VECTOR}

Kaertner in [6] provides the outline of a (numerical) algorithm for computing the FV as the persistent eigen-mode of the adjoint of the LPTV system obtained by a linearization of the system of nonlinear differential equations (DEs) (that describe the dynamics of the oscillator) around the PSSS. In [6], Kaertner uses the shooting method [7, 8] to compute the large-signal PSSS for the oscillator, followed by an eigen-decomposition of the monodromy (fundamental) matrix for the LPTV system. Kaertner observes the fact that the monodromy matrix for the LPTV system is actually computed in the course of the large-signal periodic steady-state analysis using the shooting method. The monodromy matrix for the LPTV system is the Jacobian (plus the identity matrix) of the system of nonlinear algebraic equations solved by the shooting Newton method, with the so-called phase condition removed. The monodromy matrix has one eigenvalue that is equal to exactly 1 , corresponding to the persistent oscillatory mode of the oscillator. The shooting method Jacobian matrix is equal to the monodromy matrix minus the identity matrix. Hence, it has an eigenvalue equal to 0 , meaning that it is singular. Thus, the eigenvectors corresponding to the persistent mode of the LPTV system and its adjoint lie in the null space of the shooting method Jacobian and its transpose respectively. In a non matrix-implicit implementation of the shooting method, the monodromy matrix (plus the identity matrix) is formed at the last Newton iteration in solving the shooting equations. Kaertner proposes to proceed with a full eigen-decomposition of the monodromy matrix, followed by the identification of the persistent eigen-mode. However, he does not explain how exactly this identification can be performed. The FV is then computed by traversing one period of oscillation, by effectively solving the adjoint LPTV system with the initial condition set to the persistent mode obtained from the eigen-decomposition. As a final note, Kaertner alludes to numerical problems that arise when there are other modes of the LPTV system which are very close to being persistent, meaning that they are very slowly decaying. He mentions that this may cause problems in analyzing oscillators with high-Q resonators, even though he does not offer any solutions to these numerical problems that may arise.

Anzill et. al. in [9] present an harmonic balance formulation for the oscillator noise analysis problem. Their formulation is based on linear perturbation analysis, and hence results in a non-physical and incorrect oscillator spectrum that diverges to infinity at the oscillation frequency and its harmonics [9]. The authors are aware of this problem, but they do not question the validity of the linear perturbation analysis. Instead, they portray the divergence problem only as a numerical ill-conditioning issue that causes problems when one would like to compute the noisy oscillator spectrum at frequencies close to the fundamental oscillation frequency. Then, they implicitly use the results of Floquet Theory to compute the noisy oscillator spectrum in an eigen-decomposed form, even though they never explicitly state that they are doing this. In a very clever way, they use eigen-decomposition based computations to solve the problem they have (mistakenly) 
identified as numerical ill-conditioning. Anzill et. al. in [9] observe that the FV lies in the one-dimensional null space of the transpose of the harmonic balance Jacobian matrix evaluated at the PSSS.

Demir et. al. first in $[10,11]$ and then in $[12,1,13,14]$ describe in detail a timedomain algorithm for computing the FV. Their treatment and the numerical algorithm they present is similar to Kaertner's, founded on Floquet Theory. However, they provide a detailed enough treatment of the numerical methods they propose so that a full-fledged implementation of their numerical techniques can be developed based on their thorough description. Moreover, they address the numerical problems alluded to by Kaertner in [6] regarding the existence of other closely persistent modes for the LPTV system obtained by linearization. In particular, they note the impossibility of the identification of the correct oscillatory persistent mode and hence the FV with an inspection of just the eigenvalues of the monodromy matrix. For the genuine persistent mode, the monodromy matrix has an eigenvalue equal to 1 . However, Demir et. al. observe in $[10,11]$ that, for some circuits, numerical eigen-decomposition may produce many other eigenvalues close to 1 , and that these eigenvalues may be numerically indistinguishable from the genuine eigenvalue that is supposed to be exactly at 1 . For this case, Demir et. al. [10,11] provide a simple recipe grounded on Floquet Theory for the proper resolution of the correct persistent mode, or equivalently the correct eigenvector. They observe that the inner products of the time-derivative of the periodic steady-state solution with all of the near persistent modes of the adjoint LPTV system are theoretically equal to zero, whereas the inner product with the genuine persistent mode has to be nonzero, all due to the bi-orthogonality property discussed before. Of course, due to numerical errors, this property is not exactly satisfied in practical numerical computations, but Demir et. al. $[10,11]$ claim that, with proper normalization, the comparison of the numerical values of these inner products will reveal the genuine persistent mode.

Hajimiri et. al. in [3] follow a practical and phenomenological approach to define what they call the Impulse Sensitivity Function (ISF) in order to quantify the sensitivities between the noise sources and the persisting phase fluctuations in an oscillator. Unlike the approaches described above, their formulation is not founded on Floquet Theory. However, it can be shown that the ISF defined by Hajimiri et. al is deep down, essentially equivalent to the FV defined by the Floquet Theory based approaches. Hajimiri et. al. observe that the disturbances in an oscillator result in both orbital deviations (amplitude deviations in a loose sense) that "eventually" die away and phase fluctuations that persist indefinitely even if the disturbances disappear, an observation originally made by Kaertner. Hajimiri et. al. propose two different numerical algorithms for computing the ISF. In their original proposal, Hajimiri et. al. compute the ISF by using the circuit simulator SPICE directly as a black box. They perturb the oscillator circuit in steadystate with a series of (im)pulses at time points that are swept from a reference time point in the oscillation waveform through the end of the oscillation period. After hitting the circuit with each and every one of these (im)pulses, they simulate the oscillator circuit in SPICE (transient analysis) for a "long enough" duration of time so that all of the amplitude deviations due to the impulse perturbation decay away and only the persistent phase disturbance remains. Then, they measure the amount of the persistent phase shift in the waveforms of the perturbed circuit by comparing its waveforms with an unperturbed version of the same circuit that is simulated concurrently with the perturbed one. They repeat this phase shift computation for every noise source in the circuit and for a number of pulse locations distributed evenly throughout a period of oscillation. Even though this algorithm is not very suitable for an efficient and accurate implementation for the computation of the FV, it implicitly defines ISF in such a way that ISF becomes theoretically equivalent to the FV that is defined 
based on Floquet Theory. There are various aspects (especially the "long" duration of transient analysis needed for the amplitude deviations to decay away after the circuit is hit with an impulsive perturbation) of this algorithm which make it much less efficient and also less accurate compared with algorithms based on Floquet Theory and eigendecomposed computations. Hajimiri et. al. in [3] propose a simplified, approximate algorithm for computing the ISF based on using the time-derivatives of the periodic steady-state waveforms of the oscillator, possibly motivated by the inefficiency of their original algorithm, and by the desire to obtain a manually executable, analytically calculable, simple and intuitive procedure for the computation of the ISF. However, their simple algorithm for the computation of the ISF does not produce the same results as their original algorithm based on impulsive perturbations or the other algorithms described above. This new algorithm implicitly redefines ISF, and no longer produces the sensitivity function that correctly quantifies the conversion of disturbances and noise into persistent phase fluctuations. It turns out, Kaertner, in an earlier work [5], defined a phase sensitivity function equivalent to the one that is implicitly defined by the simplified algorithm of Hajimiri. et. al. However, later in [6], Kaertner realizes that there are problems with his original definition. At the least, the FV or the ISF computed this way becomes dependent on (i.e., changes with) the scaling of the state variables that describe the oscillator. Moreover, this definition mixes persistent phase fluctuations with those that die away eventually. Kaertner fixes these problems by properly defining the FV in [6]. The simplified algorithm by Hajimiri et. al. in [3] and the algorithm by Kaertner in [5] for computing the ISF or FV would have been correct and accurate if the LPTV system obtained by the linearization of the oscillator equations at the steady-state is selfadjoint. However, this is not the case for almost all practical oscillator circuits. Still, there are some questions that have not yet been fully answered in the literature $[15,16]$. Even if the simplified algorithm by Hajimiri et. al. and the earlier algorithm by Kaertner in [5] do not produce exact quantifications for correct phase noise computations, are they "approximately" correct? If yes, is this the case for all kinds of oscillator circuits, and in general? Or for specific types of circuits, in very special cases? A thorough examination of this issue is warranted, given the popularity of the simplified algorithm for computing the ISF in the RF design community.

Demir et. al. in [4] present a novel formulation and efficient numerical method for the computation of the FV. With this new formulation, they address the problem with the correct identification of the genuine persistent eigen-mode. With their new algorithm for the computation of the FV, the identification or selection of the genuine persistent eigen-mode among the other eigen-modes becomes implicit. In [4], they observe that the FV lies in the null space of the transpose of the harmonic balance Jacobian matrix evaluated at the periodic steady-state solution. This observation forms the basis for the new formulation and the numerical method they present for the computation of the FV. Their numerical algorithm can be described, in very simple terms with a pure linear algebra perspective, as a specialized technique for the computation of a single vector that spans the one-dimensional null space of a singular matrix. This new technique is not based on eigen-decomposed computations, instead it utilizes an augmented non-singular matrix to directly solve for the single vector that spans the null space. The numerical method Demir et. al. propose in [4] relies on the harmonic balance Jacobian having a one-dimensional and well-conditioned null space, unambiguously corresponding to the persistent oscillatory mode. However, as mentioned above, there exist oscillators with many near persistent modes resulting in other near zero eigenvalues for the harmonic balance Jacobian. Moreover, the eigenvalues corresponding to the other near persistent modes may be closer to zero than the eigenvalue corresponding to the genuine persistent mode. These kinds of inaccuracies in numerical computations arise due to the truncated 
Fourier series that is used to represent the periodic quantities in harmonic balance. Similar inaccuracies also arise in time-domain computations based on shooting method formulations due to the time discretization that is used to represent the periodic signals. In fact, using truncated Fourier series in harmonic balance exactly corresponds to using a finite number of equally spaced time samples to represent a periodic waveform.

\section{SUMMARY AND CONCLUSIONS}

Based on our discussion of previous work on computational approaches to oscillator noise analysis, we conclude that the ones grounded on Floquet Theory are the most rigorous and most appropriate for the implementation of a general purpose oscillator noise analysis tool in a circuit simulator. All of the approaches based on Floquet Theory somehow try to extract or identify the persistent eigen-mode of the (adjoint) LPTV system corresponding to an eigenvalue 1 (or 0 depending on the flavor of the periodic steadystate analysis algorithm and the specific matrix being eigen-decomposed). However, all of the algorithms discussed above may run into problems when the LPTV system has other near persistent eigen-modes. Eigenvalue based selection of the genuine persistent eigen-mode fails immediately, because the real persistent mode may have an eigenvalue further away from 1 (or 0 ) compared with the other near persistent modes, due to numerical inaccuracies resulting from time discretization or the truncation of Fourier series. In our opinion, the most effective and robust method currently available for computing the FV is based on performing a full (or partial using iterative Krylov-subspace based techniques) eigen-decomposition for the transpose of either the monodromy matrix, the shooting method Jacobian or the harmonic balance Jacobian followed by an identification of the correct persistent eigen-mode by making use of the bi-orthogonality relationships discussed above. However, even this method may run into problems for some oscillators when other near persistent modes exist.

\section{REFERENCES}

1. A. Demir, A. Mehrotra, and J. Roychowdhury. Phase noise in oscillators: A unifying theory and numerical methods for characterisation. IEEE Transactions on Circuits and Systems-I: Fundamental Theory and Applications, 47(5):655, May 2000. characterisation. IEEE Transactions on Circuits and Systems-I: Fundamental Theory and Applications, 47(5):655, May 2000.
A. Demir. Phase noise and timing jitter in oscillators with colored noise sources. IEEE Transactions on Circuits and Systems-I: A. Demir. Phase noise and timing jitter in oscillators with colored noise
Fundamental Theory and Applications, 49(12):1782, December 2002.

Fundamental Theory and Applications, 49(12): 1782 , December 2002 .
A. Hajimiri and T.H. Lee. A general theory of phase noise in electrical oscillators. IEEE Journal of Solid-State Circuits, February 1998

4. A. Demir and J. Roychowdhury. A reliable and efficient procedure for oscillator ppv computation, with phase noise macromodelling applications. IEEE Transactions on Computer-Aided Design of Integrated Circuits and Systems, February 2003 .

5. F. X. Kaertner. Determination of the correlation spectrum of oscillators with low noise. IEEE Transactions on Microwave Theory and Techniques, 37(1):90-101, January 1989.

6. F. X. Kaertner. Analysis of white and $f^{-\alpha}$ noise in oscillators. International Journal of Circuit Theory and Applications, 18:485-519, 1990.

7. T.J. Aprille and T.N. Trick. Steady-state analysis of nonlinear circuits with periodic inputs. Proceedings of the IEEE, T.J. Aprille and T.N. Trick.
60(1): $108-114$, Jaunary 1972. 8. R. Telichevesky, K.S. Kundert, and J. White. Efficient steady-state analysis based on matrix-free krylov-subspace methods. In Proc. Design Automation Conference, June 1995.

9. W. Anzill and P. Russer. Determination of the correlation spectrum of oscillators with low noise. IEEE Transactions on Microwave Theory and Techniques, 41(12):2256, December 1993

10. A. Demir. Analysis and Simulation of Noise in Nonlinear Electronic Circuits and Systems. PhD thesis, University of California, Berkeley, May 1997.

11. A. Demir and A. Sangiovanni-Vincentelli. Analysis and Simulation of Noise in Nonlinear Electronic Circuits and Systems. Kluwer Academic Publishers, 1998.

12. A. Demir, A. Mehrotra, and J. Roychowdhury. Phase noise in oscillators: A unifying theory and numerical methods for characterisation. In ACM/IEEE Design Automation Conference, June 1998.

13. A. Demir Phase noise in oscillators: DAEs and colored noise sources. In IEEE/ACM International Conference on CAD, November 1998.

14. A. Demir. Floquet theory and nonlinear perturbation analysis for oscillators with differential-algebraic equations. International Journal of Circuit Theory and Applications, March-April 2000.

15. G.J. Coram. A simple 2-D oscillator to determine the correct decomposition of perturbations into amplitude and phase noise. IEEE Transactions on Circuits and Systems-I: Fundamental Theory and Applications, 48(7):896, July 2001.

16. P. Vanassche, G. Gielen, and W. Sansen. On the difference between two widely publicized methods for analyzing oscillator phase behavior. In ACM/IEEE International Conference on Computer-Aided Design, November 2002. 